\title{
A PROOF OF THE FEFFERMAN-STEIN-STRÖMBERG INEQUALITY FOR THE SHARP MAXIMAL FUNCTIONS
}

\author{
NOBUHIKO FUJII
}

(Communicated by Marshall Ash)

\begin{abstract}
We give another proof of a theorem of Strömberg for the FeffermanStein sharp maximal functions. Our method is based on a decomposition lemma which is due to the arguments of Carleson, Garnett and Jones for the functions of BMO, and it is valid for a two-weight setting under a condition which is equivalent to the $A_{\infty}$ condition in the case of equal weights.
\end{abstract}

\section{INTRODUCTION}

Let $f$ be a locally integrable function on $\mathbf{R}^{n}$ and $I$ denotes a cube in $\mathbf{R}^{n}$ with sides parallel to the axes. Then, if

$$
\sup _{I} \frac{1}{|I|} \int_{I}\left|f(x)-f_{I}\right| d x<\infty
$$

$f$ is said to be of BMO. Here $|I|$ is the Lebesgue measure of $I$ and $f_{I}=$ $1 /|I| \int_{I} f d x$. In [2] Carleson obtained a decomposition theorem for the functions of BMO, and Garnett and Jones [4] have given a new proof of the theorem of Carleson.

In both cases the methods are based on the well-known lemma of Calderón and Zygmund [1]. This lemma is also useful for locally integrable functions which are not necessarily of BMO. By using this decomposition lemma, which will appear as Lemma 1 in this note, we shall give another proof of a theorem of Strömberg [5, Theorem 3.1 . (ia), p. 523] for the Fefferman-Stein sharp maximal functions in a two-weight setting.

The sharp maximal function $f^{\#}(x)$ is defined by

$$
f^{\#}(x)=\sup \frac{1}{|I|} \int_{I}\left|f(y)-f_{I}\right| d y,
$$

where the supremum is taken over all the cubes $I$ containing $x$. (See Fefferman and Stein [3, p. 153].)

Received by the editors December 3, 1987 and, in revised form, June 12, 1988.

1980 Mathematics Subject Classification (1985 Revision). Primary 42B99.

Key words and phrases. Sharp maximal functions, $A_{\infty}$ condition. 
Let $\varphi(x)$ be a non-negative increasing function on $[0, \infty]$ satisfying that $\varphi(0)=0$ and

$$
\varphi(2 t) \leq C_{0} \varphi(t) \quad \text { for any } t>0,
$$

where the constant $C_{0}$ is independent of $t$. For example, the functions $\varphi(t)=$ $t^{p}, 0<p<\infty$, satisfy (1.1).

The growth condition (1.1) for $\varphi$ was introduced into sharp maximal function inequalities by Strömberg [5, p. 512].

Now we state our result:

Theorem. Let $w(x)$ and $v(x)$ be non-negative and locally integrable functions on $\mathbb{R}^{n}$. Suppose there exist positive numbers $C_{1}, \alpha$ and $\beta$ satisfying that $0<\alpha<1$, and that for any cube $I$ and a measurable subset $E$ of $I$

$$
\int_{E} w(x) d x \leq C_{1}\left(\frac{|E|}{|I|}\right)^{\beta} \int_{I \backslash E} v(x) d x
$$

whenever $|E| \leq \alpha|I|$.

Then there exists a positive constant $C_{2}$, depending only on $C_{0}, C_{1}, \alpha, \beta$ and the dimension $n$, which satisfies that

$$
\int_{\mathbf{R}^{n}} \varphi(|f(x)|) w(x) d x \leq C_{2} \int_{\mathbf{R}^{n}} \varphi\left(f^{\#}(x)\right) v(x) d x,
$$

for any locally integrable function $f$, provided that

$$
\lim _{|Q| \rightarrow \infty} f_{Q}=0 \text {, }
$$

where $Q$ are the cubes centered at the origin.

Remark 1. By Lebesgue's theorem on differentiating the integral, (1.2) implies that

$$
w(x) \leq C_{3} v(x) \quad \text { for almost all } x \in \mathbb{R}^{n} .
$$

Remark 2. When $w=v$, the condition (1.2) is equivalent to the $A_{\infty}$ condition:

$$
\int_{E} w d x \leq C\left(\frac{|E|}{|I|}\right)^{\beta} \int_{I} w d x
$$

for a cube $I$ and a subset $E$ of $I$. In this case the theorem may be proved by the methods of Fefferman and Stein [3, pp. 153-155] and Strömberg [5, pp. 524-525].

We give a simple example of a pair $(w, v)$ of functions on $\mathbb{R}$ which satisfies (1.2). We define that $w(x)=0$ if $0 \leq x<1$ and $w(x)=1$ otherwise, and that $v(x)=0$ if $\frac{1}{3} \leq x<\frac{2}{3}$ and $v(x)=1$ otherwise. Then we can easily see that (1.2) holds for $w$ and $v$, but neither of them is an $A_{\infty}$ weight and we cannot also insert any $A_{\infty}$ weight between them. 
Throughout this note various constants $C$ and $C_{j}$ depend only on $C_{0}, C_{1}$, $\alpha, \beta$, and $n$.

\section{PROOF OF THEOREM}

We shall begin by stating the lemma. Let $f$ be a locally integrable function on $\mathbb{R}^{n}$ and let $Q$ be a cube in $\mathbb{R}^{n}$. We put for $x$ in $Q$

$$
f^{\#, Q}(x)=\sup \frac{1}{|I|} \int_{I}\left|f(y)-f_{I}\right| d y
$$

where the supremum is taken over all the dyadic subcubes $I$ of $Q$ which contain $x$. Dyadic subcubes $I$ of $Q$ with measure $2^{-j n}|Q|$ are obtained by bisecting each side of $Q j$ times, respectively.

Lemma 1. Let $A$ be a positive number and $f$ be a locally integrable function. If $\left|\left\{x \in Q ;\left|f(x)-f_{Q}\right|>A f^{\#, Q}(x)\right\}\right| \neq 0$ then there exist a measurable function $g$, a sequence $\left\{a_{\nu}^{(j)}\right\}_{j, \nu}$ of nonzero numbers and families of dyadic subcubes $\mathscr{F}_{j}=\left\{I_{\nu}^{j}\right\}_{\nu}, j=1,2 \ldots$, in $Q$ satisfying that

(i) the cubes $I_{\nu}^{j}$ in $\mathscr{F}_{j}$ are pairwise disjoint for each $j$,

(ii) $f(x)=f_{Q}+g(x)+\sum_{j=1}^{\infty} \sum_{\nu} a_{\nu}^{(j)} \chi_{I_{\nu}^{j}}(x)$ for almost all $x$ in $Q$ where $\chi_{I_{\nu}^{j}}(x)$ is the characteristic function of $I_{\nu}^{j}$,

(iii) $|g(x)| \leq A f^{\#, Q}(x)$ for almost all $x$ in $Q$,

(iv) $\left|a_{\nu}^{(j)}\right| \leq\left(2^{n}+A\right) \sup _{I \supseteq I_{\nu}^{j}} \frac{1}{|I|} \int_{I}\left|f-f_{I}\right| d x$,

where $I$ are dyadic subcubes of $Q$, and

(v) $\bigcup_{I_{\mu}^{j+1} \in \mathscr{F}_{j+1}} I_{\mu}^{j+1} \subset \bigcup_{I_{\nu}^{j} \in \mathscr{F}_{j}} I_{\nu}^{j}$ for every $j$,

moreover, if $k>j$, then for $I_{\nu}^{j} \in \mathscr{F}_{j}$

$$
\sum_{I_{\mu}^{k} \subset I_{\nu}^{j}, I_{\mu}^{k} \in \mathscr{F}_{k}}\left|I_{\mu}^{k}\right| \leq A^{-(k-j)}\left|I_{\nu}^{j}\right| .
$$

Remark 3. When $f$ is of BMO, Lemma 1 means Theorem 2.1 of Garnett and Jones [4, pp . 355-356]. And our proof of Lemma 1 will be the same as that of Theorem 2.1 of Garnett and Jones in that case. So we shall sketch the proof of Lemma 1.

Proof of Lemma 1. We shall use the manner of Carleson in [2, pp. 273-276].

Let $\left\{Q_{\nu}^{j}\right\}_{\nu=1}^{2^{j n}}$ be the family of all the dyadic subcubes of $Q$ with measure $2^{-j n}|Q|$.

Put $f_{1}(x)=f(x)-f_{Q}$. Inductively for every $j \geq 1$, we set

$$
b_{\nu}^{(j)}=\left(f_{j}\right)_{Q_{\nu}^{j}} \quad \text { if }\left|\left(f_{j}\right)_{Q_{\nu}^{j}}\right|>A \sup _{Q_{\mu}^{k} \supset Q_{\nu}^{j}} \frac{1}{\left|Q_{\mu}^{k}\right|} \int_{Q_{\mu}^{k}}\left|f(x)-f_{Q_{\mu}^{k}}\right| d x
$$

and $b_{\nu}^{(j)}=0$ otherwise and we set $f_{j+1}(x)=f_{j}(x)-\sum_{\nu} b_{\nu}^{(j)} \chi_{Q_{\nu}^{j}}(x)$. 
Then

$$
\left|\left(f_{j}\right)_{Q_{\mu}^{j-1}}\right| \leq A \sup _{Q_{\xi}^{k} \supset Q_{\mu}^{j-1}}\left(\left|f-f_{Q_{\xi}^{k}}\right|\right)_{Q_{\xi}^{k}}
$$

and therefore, if $Q_{\nu}^{j} \subset Q_{\mu}^{j-1}$,

$$
\begin{aligned}
\left|b_{\nu}^{(j)}\right| & \leq\left|\left(f_{j}\right)_{Q_{\nu}^{j}}-\left(f_{j}\right)_{Q_{\mu}^{j-1}}\right|+\left|\left(f_{j}\right)_{Q_{\mu}^{j-1}}\right| \\
& \leq\left(2^{n}+A\right) \sup _{Q_{\xi}^{k} \supset Q_{\mu}^{j-1}}\left(\left|f-f_{Q_{\xi}^{k}}\right|\right)_{Q_{\xi}^{k}} .
\end{aligned}
$$

Lebesgue's theorem on differentiating the integral shows that

$$
g(x)=\lim _{j \rightarrow \infty} f_{j}(x)
$$

converges for almost all $x$ in $Q$ and (2.1) implies (iii).

Let $\mathscr{F}_{1}=\left\{Q_{\nu}^{k} ; b_{\nu}^{(k)} \neq 0\right.$ and maximal $\}$ and inductively we let $\mathscr{F}_{j}=\left\{Q_{\nu}^{k} ; b_{\nu}^{(k)}\right.$ $\neq 0, Q_{\nu}^{k} \notin \mathscr{F}_{j-1}$ and maximal $\}$. If $\mathscr{F}_{j} \neq \varnothing$, we set $a_{\nu}^{(j)}=b_{\nu}^{(k)} \neq 0$ and $I_{\nu}^{j}=Q_{\nu}^{k}$ for $Q_{\nu}^{k}$ in $\mathscr{F}_{j}$. Then (2.2) implies (iv).

If $I_{\mu}^{j+1} \subset I_{\nu}^{j}$, then $\left|a_{\mu}^{(j+1)}\right|=\left|f_{I_{\mu}^{j+1}}-f_{I_{\nu}^{j}}\right|>A\left(\left|f-f_{I_{\nu}^{j}}\right|\right)_{I_{\nu}^{j}}$. Hence

$$
\left(\left|f-f_{I_{\nu}^{\prime}}\right|\right)_{I_{\nu}^{j}}<\frac{1}{A}\left(\left|f-f_{I_{\nu}^{\prime}}\right|\right)_{I_{\mu}^{j+1}} .
$$

Summing up (2.3) with respect to $I_{\mu}^{j+1} \subset I_{\nu}^{j}$ we get

$$
\sum_{I_{\mu}^{j+1} \subset I_{\nu}^{\prime}}\left|I_{\mu}^{j+1}\right| \leq \frac{1}{A}\left|I_{\nu}^{j}\right|
$$

By repeating this argument we have $(\mathrm{v})$.

We shall also need later the following simple lemma:

Lemma 2. Let $\left\{a^{(j)}\right\}_{j=1}^{k}$ be a sequence of numbers. Then

$$
\varphi\left(\sum_{j=1}^{k}\left|a^{(j)}\right|\right) \leq \sum_{j=1}^{k} C_{0}^{k-j+1} \varphi\left(\left|a^{(j)}\right|\right),
$$

where $C_{0}$ is the constant which satisfies (1.1).

The conclusion of Lemma 2 is immediate from the fact that

$$
\varphi(|a+b|) \leq C_{0}\{\varphi(|a|)+\varphi(|b|)\} .
$$

Proof of Theorem. Take $A$ to be a positive number larger than $\max \left\{1 / \alpha, C_{0}^{1 / \beta}\right\}$, and let $Q$ be a cube centered at the origin. Then, by Lemma 1 for the number $A$ and the cube $Q$ we have a function $g(x)$, a sequence $\left\{a_{\nu}^{(j)}\right\}$ of numbers and families $\mathscr{F}_{j}$ of dyadic subcubes of $Q$ which satisfy (i), (ii), (iii), (iv), and (v) of Lemma 1. (If $\left|\left\{x \in Q ;\left|f(x)-f_{Q}\right|>A f^{\#, Q}(x)\right\}\right|=0$, we can put $f(x)=f_{Q}+g(x)$, where $g$ satisfies (iii).) 
If a point $x$ in $Q$ satisfies (ii) and if $|f(x)| \geq 2\left|f_{Q}\right|$, we get

$$
|f(x)| \leq 2\left|g(x)+\sum_{j, \nu} a_{\nu}^{(j)} \chi_{I_{\nu}^{j}}(x)\right| .
$$

Therefore, we have from (1.1)

$$
\begin{gathered}
\int_{Q \cap\left\{|f(x)| \geq 2\left|f_{Q}\right|\right\}} \varphi(|f(x)|) w(x) d x \\
\leq C_{0} \int_{Q} \varphi\left(\left|g(x)+\sum_{j, \nu} a_{\nu}^{(j)} \chi_{I_{\nu}^{j}}(x)\right|\right) w(x) d x \\
\leq C_{0}^{2}\left\{\int_{Q} \varphi(|g(x)|) w(x) d x+\int_{Q} \varphi\left(\sum_{j, \nu}\left|a_{\nu}^{(j)}\right| \chi_{I_{\nu}^{j}}(x)\right) w(x) d x\right\} .
\end{gathered}
$$

Using (iii), (1.1) and (1.5) we obtain

$$
\int_{Q} \varphi(|g(x)|) w(x) d x \leq C_{3} C_{0}^{\log A} \int_{Q} \varphi\left(f^{\#, Q}(x)\right) v(x) d x .
$$

Next we shall show the following estimate:

$$
\int_{Q} \varphi\left(\sum_{j=1}^{\infty} \sum_{\nu}\left|a_{\nu}^{(j)}\right| \chi_{I_{\nu}^{j}}(x)\right) w(x) d x \leq C_{4} \int_{Q} \varphi\left(f^{\#, Q}(x)\right) v(x) d x .
$$

From ( $v$ ) we can divide the integration domain of the left-hand side of (2.6) as follows:

$$
\begin{aligned}
& \int_{Q} \varphi\left(\sum_{j=1}^{\infty} \sum_{\nu}\left|a_{\nu}^{(j)}\right| \chi_{I_{\nu^{j}}}\right) w d x \\
& =\int_{\underset{\mu}{U I_{\mu}^{\prime} \backslash \cup I_{\xi}^{2}}} \varphi\left(\sum_{\nu}\left|a_{\nu}^{(1)}\right| \chi_{I_{\nu}^{\prime}}\right) w d x \\
& +\sum_{k=2}^{\infty} \int_{\underset{\mu}{\cup I_{\mu}^{k} \backslash \bigcup \underset{\xi}{\xi_{k}} I^{k+1}}} \varphi\left(\sum_{j=1}^{k} \sum_{\nu}\left|a_{\nu}^{(j)}\right| \chi_{I_{\nu}^{j}}\right) w d x,
\end{aligned}
$$

where $I_{\mu}^{k} \in \mathscr{F}_{k}$ and $I_{\xi}^{k+1} \in \mathscr{F}_{k+1}$. For every $k \geq 2$ we observe

$$
\begin{aligned}
& \int_{\underset{\mu}{\cup I_{\mu}^{k} \backslash \bigcup_{\xi} I_{\xi}^{k+1}}} \varphi\left(\sum_{j=1}^{k} \sum_{\nu}\left|a_{\nu}^{(j)}\right| \chi_{I_{\nu}^{\prime}}\right) w d x
\end{aligned}
$$

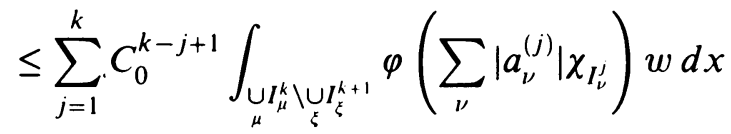


(by Lemma 2)

$$
\begin{aligned}
\leq & C_{0} \int_{\underset{\mu}{\cup I_{\mu}^{k} \backslash \bigcup_{\xi} I_{\xi}^{k+1}}} \varphi\left(\sum_{\nu}\left|a_{\nu}^{(k)}\right| \chi_{I_{\nu}^{k}}\right) w d x \\
& +\sum_{j=1}^{k-1} C_{0}^{k-j+1} \int_{\cup_{\mu} I_{\mu}^{k}} \varphi\left(\sum_{\nu}\left|a_{\nu}^{(j)}\right| \chi_{I_{\nu}^{j}}\right) w d x .
\end{aligned}
$$

From (v) and the hypothesis (1.2) it follows that

$$
\begin{aligned}
& \int_{\cup_{\mu} I_{\mu}^{k}} \varphi\left(\sum_{\nu}\left|a_{\nu}^{(j)}\right| \chi_{I_{\nu}^{j}}\right) w d x \\
& \leq C_{1} A^{-\beta(k-j)} \int_{\underset{\nu}{I_{\nu}^{j} \backslash \cup_{\mu}^{k}}} \varphi\left(\sum_{\nu}\left|a_{\nu}^{(j)}\right| \chi_{I_{\nu}^{j}}\right) v d x .
\end{aligned}
$$

And from (1.5) we have for $k \geq 2$ and also for $k=1$

$$
\begin{aligned}
& \int_{\mu}^{\cup I_{\mu}^{k} \backslash \cup_{\xi} I_{\xi}^{k+1}} \varphi\left(\sum_{\nu}\left|a_{\nu}^{(k)}\right| \chi_{I_{\nu}^{k}}\right) w d x \\
& \leq C_{3} \int_{\underset{\mu}{\cup} I_{\mu}^{k} \backslash \bigcup_{\xi} I_{\xi}^{k+1}} \varphi\left(\sum_{\nu}\left|a_{\nu}^{(k)}\right| \chi_{I_{\nu}^{k}}\right) v d x .
\end{aligned}
$$

Therefore, we see that the right-hand side of (2.8) is majorized by

$$
\begin{aligned}
& C_{5}\left\{\int_{\underset{\mu}{\cup I_{\mu}^{k} \backslash \cup I_{\xi}^{k+1}}} \varphi\left(\sum_{\nu}\left|a_{\nu}^{(k)}\right| \chi_{I_{\nu}^{k}}\right) v d x\right. \\
& \left.\quad+C_{1} \sum_{j=1}^{k-1}\left(C_{0} A^{-\beta}\right)^{k-j} \int_{\underset{\nu}{\cup I_{\nu}^{j} \backslash \cup I_{\mu}^{k}}} \varphi\left(\sum_{\nu}\left|a_{\nu}^{(j)}\right| \chi_{I_{\nu}^{j}}\right) v d x\right\} .
\end{aligned}
$$

Summing up the above expression with respect to $k$ and using (iv) we see that the left-hand side of (2.7) is majorized by

$$
\begin{gathered}
C_{5}\left\{\sum_{k=1}^{\infty} \int_{\underset{\mu}{\cup} I_{\mu}^{k} \backslash \cup I_{\xi}^{k+1}} \varphi\left(\sum_{\nu}\left|a_{\nu}^{(k)}\right| \chi_{I_{\nu}^{k}}\right) v d x\right. \\
\left.+C_{1} \sum_{k=2}^{\infty} \sum_{j=1}^{k-1}\left(C_{0} A^{-\beta}\right)^{k-j} \int_{\underset{\nu}{\cup I_{\nu}^{j} \backslash \bigcup_{\mu} I_{\mu}^{k}}} \varphi\left(\sum_{\nu}\left|a_{\nu}^{(j)}\right| \chi_{I_{\nu}^{j}}\right) v d x\right\} \\
\leq C_{6}\left\{\int_{Q} \varphi\left(f^{\#, Q}(x)\right) v d x\right. \\
\left.+C_{1} \sum_{k=2}^{\infty} \sum_{j=1}^{k-1}\left(C_{0} A^{-\beta}\right)^{k-j} \int_{\underset{\nu}{\cup I_{\nu}^{j} \backslash \cup \cup} I_{\mu}^{k}} \varphi\left(f^{\#, Q}(x)\right) v d x\right\} .
\end{gathered}
$$


When we interchange the order of summation, the second term of the last expression becomes

$$
\begin{aligned}
& C_{1} \sum_{j=1}^{\infty} \sum_{k=j+1}^{\infty}\left(C_{0} A^{-\beta}\right)^{k-j} \int_{\bigcup_{\nu} I_{\nu}^{j} \backslash \cup I_{\mu}^{k}} \varphi\left(f^{\#, Q}(x)\right) v d x \\
& =C_{1} \sum_{j=1}^{\infty} \sum_{l=1}^{\infty}\left(C_{0} A^{-\beta}\right)^{l} \int_{\underbrace{}_{\nu} I_{\nu}^{j} \backslash \bigcup_{\mu} I_{\mu}^{l+j}} \varphi\left(f^{\#, Q}(x)\right) v d x \\
& =C_{1} \sum_{l=1}^{\infty}\left(C_{0} A^{-\beta}\right)^{l} \sum_{j=1}^{\infty} \int_{\bigcup_{\nu} I_{\nu}^{j} \backslash \cup \bigcup_{\mu} I_{\mu}^{l+j}} \varphi\left(f^{\#, Q}(x)\right) v d x .
\end{aligned}
$$

Since the integration domains $\left\{\cup_{\nu} I_{\nu}^{j} \backslash \cup_{\mu} I_{\mu}^{l+j}\right\}_{j}$ are overlapping at most $l$ times for each $l$, the above expression is bounded by

$$
C_{1} \sum_{l=1}^{\infty} l\left(C_{0} A^{-\beta}\right)^{l} \int_{Q} \varphi\left(f^{\#, Q}(x)\right) v d x .
$$

Because $C_{0} A^{-\beta}<1$ we conclude that (2.9) is majorized by $C \int_{Q} \varphi\left(f^{\#, Q}(x)\right) v d x$.

Thus we get (2.6). And (2.4), (2.5), and (2.6) imply that

$$
\int_{Q \cap\left\{|f(x)| \geq 2\left|f_{Q}\right|\right\}} \varphi(|f(x)|) w d x \leq C_{7} \int_{\mathbf{R}^{n}} \varphi\left(f^{\#}(x)\right) v d x .
$$

When $|Q| \rightarrow \infty$, we obtain the conclusion (1.3) of the theorem by the hypothesis (1.4). This completes the proof of the theorem.

I would like to add that if we replace $f_{Q}$ by the median value $m_{f}(Q)$, then the theorem holds for the sharp maximal functions $M_{0,1 / A}^{\#} f(x)$, where $A$ is sufficiently large.

\section{ACKNOWLEDGMENTS}

I would like to thank the referee for his careful reading and suggestions, and also thank the graduate students of the Department of Mathematics at Tokyo Metropolitan University for their help and kindness.

\section{REFERENCES}

1. A. P. Calderón and A. Zygmund, On the existence of certain singular integrals, Acta Math. 88 (1952), 85-139.

2. L. Carleson, Two remarks on $H^{1}$ and BMO, Adv. in Math. 22 (1976), 267-277.

3. C. Fefferman and E. M. Stein, $H^{p}$ spaces of several variables, Acta Math. 129 (1972), 137-193.

4. J. B. Garnett and P. W. Jones, BMO from dyadic BMO, Pacific J. Math. 99 (1982), 351-371.

5. J.-O. Strömberg, Bounded mean oscillation with Orlicz norms and duality of Hardy spaces, Indiana Univ. Math. J. 28 (1979), 511-544.

Tokai University, HiRatsuka, Kanagawa 259-12, Japan

Current address: 3-4-12 Yurigaoka, Asao-ku, Kawasaki, Kanagawa 215, Japan 